\title{
On the dimension of splines spaces over T-meshes with smoothing cofactor-conformality method
}

\author{
Xin Li Jiansong Deng \\ University of Science and Technology of China, Hefei, Anhui, P. R. China
}

\begin{abstract}
This paper provides a general formula for the dimension of spline space over general planar T-meshes (having concave corners or holes) by using the smoothing cofactor-conformality method. We introduce a new notion, the diagonalizable T-mesh, where the dimension formula is only associated with the topological information of the T-mesh. A necessary and sufficient condition for characterization the diagonalizable T-mesh is also provided. By this new notion, we obtain some new dimension results for the spline spaces over T-meshes.
\end{abstract}

Key words: Dimension, T-splines, Spline space over T-mesh, smoothing cofactor-conformality

\section{Introduction}

NURBS (Non-Uniform Rational B-Spline) is the de facto standard that is used to generate and represent free-form curves and surfaces in CAD [1]. It is also a desirable tool for isogeometric analysis [2]. A well-known and significant disadvantage of NURBS is that it is based on a tensor-product structure that uses a global knot insertion operation. It is necessary to generalize NURBS space to spline space which can handle T-junctions, or hanging nodes.

Many researchers have attempted on this issue and several different methods have been developed over the years, including Hierarchical B-splines [3, 4, 5, 6], T-splines [7, 8, 9], Polynomial Splines over T-meshes [10, 11], and LR B-splines [12, 13]. In order to apply the above locally refinable splines to isogeometric analysis [14], a very important topic is to unravel the corresponding spline spaces which have important implications in establishing approximation, stability, and error estimates [15]. For example, [16] studies the completeness of hierarchical B-spline space by calculating the dimension of spline space over some hierarchical T-meshes. Analysis-suitable T-spline spaces $[9,17,18]$ is discovered by using the dimension result of spline space over another T-mesh. The LR B-spline space is characterized according to the dimension formula in [13]. Thus, in order to understand the locally refinable spline spaces, one common, foundational, but non-trivial step is to calculate the dimension of the spline space over some T-meshes.

There are several different approaches to calculate the dimension of the spline space over some T-meshes, including the B-net method [10], the minimal determining set method [19], the smoothing cofactor-conformality method [20] and the homological technique [13]. The present paper focuses on the smoothing cofactor-conformality method. Because the smoothing cofactor-conformality method can convert the smoothness conditions into algebraic forms, we directly focus on the algebraic forms and study the matrix. If the matrix can be formed into a block upper triangular matrix, the dimension of the linear system is not associated with the knot values. Then, we use the condition to find a new notion, (diagonalizable T-mesh), regarding the corresponding T-meshes. A similar notion, (weighted 
T-mesh, definition 3.8 in [13]), is independently discovered in [13], where the dimension (Theorem 3.3 and 3.9) is able to be computed in an explicit formula by using the homological techniques. Although the weighted T-mesh is associated with a predefined order for the l-edges, it can also be generalized to the T-mesh when the order for the l-edges exists, which is the same as the diagonalizable T-mesh in the present paper. The other difference of the present paper is that we provide a necessary and sufficient condition for characterization a diagonalizable T-mesh, which can be used to find some new results for the spline space with reduced smoothness [10,21], and when the T-mesh has a sufficient amount of mono-vertices (the definition is in section 2). The present paper is also a generalization of the first dimension of spline space over T-mesh paper with smoothing cofactor-conformality method [20]. There are two main difference between these two papers. Firstly, we generalize the result to handle more general type of T-meshes. Secondly, the notion of diagonalizable T-mesh doesn't require the pre-defined order of l-edges while the papers [20,22] are based on the predefined order of l-edges. In summary, the main contributions of the present paper include,

- We provide a general formula for the dimension of the spline space over the T-mesh (Theorem 3.1 and 3.3$)$;

- We provide a new notion, the diagonalizable T-mesh, where the dimension formula is only associated with the topological information of the T-mesh. We also provide a necessary and sufficient condition that is required characterize diagonalizable T-meshes (Theorem 4.4 and 4.5);

- We provide new dimension results for the spline space over T-meshes that do not have a nested structure (in Section 5).

\subsection{Prior work}

Locally refinable splines. Four typical approaches have developed to generalize NURBS space to spline space which can handle T-junctions: the hierarchical B-splines, the T-splines, the splines over T-meshes and the LR B-splines. The hierarchical B-splines are originally introduced in 1988 by Forsey and Bartels [3], which can be locally refined by using overlays. Then, Kraft suggests a selection mechanism for hierarchical B-splines [4] to ensure their linear independence. Kraft's construction is further elaborated in $[5,6]$ for the application in isogeometric analysis. T-splines are collections of B-spline functions that are defined on a T-mesh (T-grid) [7, 8]. T-splines are compatible forwards and backwards with NURBS and they have several advantages over NURBS, including local refinement $[8,17]$ and watertightness [23]. These capabilities make T-splines attractive for both CAD and isogeometric analysis [14]. Spline space over a T-mesh, or $\mathcal{S}\left(d_{1}, d_{2}, \alpha, \beta, \mathcal{T}\right)$, is first introduced in [10], which is a bi-degree $\left(d_{1}, d_{2}\right)$ piecewise polynomial spline space over T-mesh $\mathcal{T}$ with smoothness orders $\alpha$ and $\beta$ in two directions. Later, this spline is applied in adaptive fitting [11], stitching [24], simplification [25], and isogeometric analysis [26, 27], as well as for solving elliptic equations [28]. The concept is also generalized to spline space over triangulations with hanging nodes [29]. Locally refinable splines (LR-splines) are proposed by Dokken et al. [12], with the refining process based on hand-in-hand LR-refinement which starts from a tensor-product mesh.

Dimension of spline spaces over T-meshes. In 2006, [10] studies the dimension of the spline space under certain constraint that the order of smoothness is less than half of the degree of the spline functions. If the T-mesh has no cycles, [10] provides the explicit dimension formula. [21] and [20] also give the result by using the minimal determining set method and the smoothing cofactor-conformality method respectively. Later, [30] analyzes a special T-spline with reduced smoothness by using the 
dimension formula in [10]. In 2011, [31] discovers that the dimension of the associated spline space is instable over particular T-meshes, (i.e, the dimension is not only associated with the topological information of the T-mesh but it is also associated with the geometric information of the T-mesh). Additionally, in [32], D. Berdinsky et al. provide two more examples of spline spaces $\mathcal{S}(5,5,3,3, \mathscr{T})$ and $\mathcal{S}(4,4,2,2, \mathscr{T})$ regarding the instability of the dimension. [13] provides a general formula for the spline spaces by using the homological techniques. Unfortunately there is a term in the dimension formula that is very difficult to compute in practice. Thus, they define a class of T-meshes, the weighted T-meshes, over which the dimension can be computed in an explicit formula. The weighted T-mesh is associated with the order of the l-edges. [33] provides the dimension for spline space $\mathcal{S}(d, d, d-1, d-1, \mathcal{T})$ over a special hierarchical T-mesh by using the homological algebra technique. [34, 35] derives a dimension formula for $C^{1}$ biquadratic and $C^{2}$ bi-cubic spline spaces over hierarchical T-meshes. [16] provides certain conditions that were necessary for the hierarchical spline basis to span the entire space of the piecewise polynomial functions defined on the underlying grid with a given degree and smoothness.

\subsection{Organization}

The remainder of the paper is structured as follows. Pertinent background information regarding the spline space over T-mesh is reviewed in Section 2. In Section 3, we review the smoothing cofactorconformality method to analyze the dimension of the spline space over T-meshes. In section 4, we provide a condition under which we can explicitly calculate dimension formula for the spline space over the T-mesh. In section 5, we provide new dimension results for the spline space over T-meshes that do not have a nested structure. The last section discusses our conclusion and future work.

\section{The T-mesh and the spline over T-mesh}

In this section, we briefly review the notions of the T-mesh and the spline space over a T-mesh.

\subsection{T-mesh}

A T-mesh $\mathcal{T}$ is a collection of all of the elements of axis-aligned rectangles $F_{i}$ such that the interior of the domain $\Omega$ is $\cup F_{i}$, and the distinct rectangles $F_{i}$ and $F_{j}$ can only intersect at points on their edges. In the present paper, we also require the T-meshes to be regular. Here, regular means that for any vertex in the T-mesh, the set of all of the rectangles that contain the vertex has a connected interior [21]. For example, in Figure 1, T-mesh b is a regular T-mesh but T-mesh $\mathrm{c}$ is not a regular T-mesh because the red vertex $v$ doesn't satisfy the requirement for a regular T-mesh. The rectangles $F_{i}$ are also called the faces or cells of the T-mesh. The vertices of the rectangles are called the nodes or vertices for a T-mesh. The line segment that contains two adjacent vertices on a grid line is called an edge of the T-mesh. T-meshes include tensor-product meshes as a special case. However, in contrast to tensor-product meshes, T-meshes are allowed to have T-junctions, or T-nodes, which are the vertices of one rectangle that lie in the interior of an edge of another rectangle. The domain $\Omega$ does not need to be rectangular, so it may have concave corners and holes (the grey region in Figure 1).

If a vertex is on the boundary grid line of the T-mesh, it is called a boundary vertex. Otherwise, it is called an interior vertex. For example, in the T-mesh a in Figure 1, vertex $V_{58}$ is an interior vertex, $V_{15}, V_{16}$ and $V_{57}$ are boundary vertices. An edge is called a boundary edge if it is part of exactly one face; otherwise, it is called an interior edge. If an interior edge has at least one boundary vertex as an end vertex, the edge is called a neighbor-boundary edge. A composite edge (or c-edge) is a line segment that consists of one or more edges. It is the longest possible line segment where the interior vertices 

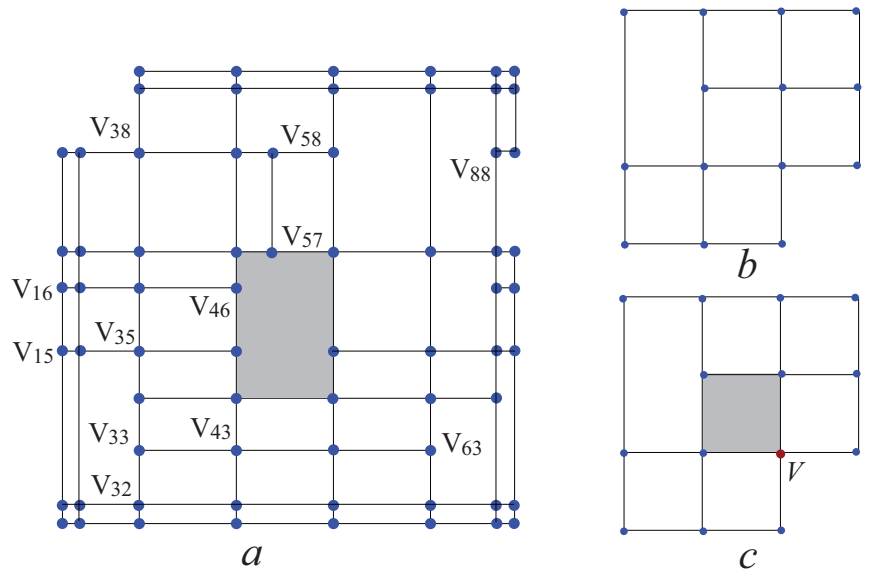

Figure 1: Example T-meshes.

are all T-junctions. An l-edge is a line segment which consists of several interior edges. It is the longest possible line segment, where all the interior edges are connected, and the two end points are T-junctions or boundary vertices. If the two end vertices of an l-edge are interior vertices, the l-edge is called an interior l-edge. For example, in the T-mesh a in Figure $1, V_{33} V_{43}, V_{57} V_{58}$ are c-edges while $V_{57} V_{58}$, $V_{33} V_{63}$ are l-edges. $V_{33} V_{63}$ is an interior l-edges and edge $V_{57} V_{58}$ is a neighbor-boundary edge. If two end vertices of a l-edge are both boundary vertices, the l-edge is called a cross-cut. Otherwise, if one end vertex is a boundary vertex and the other end vertex is an interior vertex, the l-edge is called a ray. For example, in the T-mesh a in Figure 1 , the l-edge $V_{16} V_{46}$ is a cross-cut, while $V_{57} V_{58}$ is a ray. A mono-vertex is the intersection between an interior l-edge and a cross-cut or a ray, and a free-vertex is the intersection between cross-cuts and rays.

Definition 2.1. Suppose $T_{1}, \ldots, T_{n}$ is a collection of T-nodes in a T-mesh such that for each $i=$ $1, \ldots, n$, the vertex $T_{i}$ lies in the interior of a c-edge with one endpoint at $T_{i+1}$, where we set $T_{n+1}=T_{1}$. Then, we say that $T_{1}, \ldots, T_{n}$ form a c-cycle (this is the definition of the cycle in [21]).

Definition 2.2. Suppose $T_{1}, \ldots, T_{n}$ is a collection of T-nodes in a T-mesh such that for each $i=$ $1, \ldots, n$, the vertex $T_{i}$ lies in the interior of an l-edge with one endpoint at $T_{i+1}$, where we set $T_{n+1}=T_{1}$. Then we say that $T_{1}, \ldots, T_{n}$ form an l-cycle.

It is evident that if a T-mesh has no l-cycles, then it has no c-cycles. However, in contrast, this is not correct (see the T-mesh in Figure 8 as an example, which has no c-cycles, but has an l-cycle). Now, we introduce some more notations for a T-mesh in Table 1 that will be used later.

\subsection{The spline space over a T-mesh}

Given a T-mesh $\mathcal{T} \in \mathbb{R}^{2}$, let $\mathcal{F}$ denote all of the cells in $\mathcal{T}$ and $\Omega$ be the region that is occupied by all of the cells in $\mathcal{T}$. The bi-degree $\left(d_{1}, d_{2}\right)$ polynomial spline space over a $\mathrm{T}$-mesh $\mathcal{T}$ with smoothness order $\alpha$ and $\beta$ is defined as

$$
\mathcal{S}\left(d_{1}, d_{2}, \alpha, \beta, \mathcal{T}\right):=\left\{f(x, y) \in C^{\alpha, \beta}(\Omega)|f|_{\phi} \in \mathrm{P}_{d_{1} d_{2}}, \forall \phi \in \mathcal{F}\right\}
$$

where $\mathrm{P}_{d_{1} d_{2}}$ is the space of all of the polynomials with bi-degree $\left(d_{1}, d_{2}\right)$, and $C^{\alpha, \beta}(\Omega)$ is the space that consists of all of the bivariate functions that are continuous in $\Omega$, with order $\alpha$ along $x$ direction and 
Table 1: Notations for a T-mesh

\begin{tabular}{ll}
\hline$F$ & number of faces in $\mathcal{T}$ \\
$E^{h}$ & number of horizontal interior edges in $\mathcal{T}$ \\
$E^{v}$ & number of vertical interior edges in $\mathcal{T}$ \\
$V$ & number of interior vertices in $\mathcal{T}$ \\
$C^{h}$ & number of horizontal cross-cuts in $\mathcal{T}$ \\
$C^{v}$ & number of vertical cross-cuts in $\mathcal{T}$ \\
$T^{h}$ & number of horizontal interior l-edges in $\mathcal{T}$ \\
$T^{v}$ & number of vertical interior l-edges in $\mathcal{T}$ \\
$n_{e}$ & number of interior l-edges in $\mathcal{T}\left(T^{h}+T^{v}\right)$ \\
$V^{+}$ & number of free-vertices in $\mathcal{T}$ \\
$N^{h}$ & minimal integer larger than or equal to $\frac{d_{1}+1}{d_{1}-\alpha}$ \\
$N^{v}$ & minimal integer larger than or equal to $\frac{d_{2}+1}{d_{2}-\beta}$ \\
$n_{c}$ & $\left(d_{1}-\alpha\right)\left(d_{2}-\beta\right)\left(V-V^{+}\right)$ \\
$n_{r}$ & $\left(d_{1}+1\right)\left(d_{2}-\beta\right) T^{h}+\left(d_{2}+1\right)\left(d_{1}-\alpha\right) T^{v}$ \\
\hline
\end{tabular}

with order $\beta$ along $y$ direction. It is obvious that $\mathcal{S}\left(d_{1}, d_{2}, \alpha, \beta, \mathcal{T}\right)$ is a linear space, which is called the spline space over the given T-mesh $\mathcal{T}$.

\section{The smoothing cofactor-conformality method}

In this section, we review the smoothing cofactor-conformality method that was introduced in [36] and [37] to compute the dimension of the spline space over T-meshes. In regard to the theory of multivariate splines, to calculate the dimension of a spline space, one first transfers the smoothness conditions into algebraic forms.

\subsection{Cofactors}

Referring to Figure 2, for any interior vertex $v_{i, j}=\left(x_{i}, y_{j}\right)$, suppose the four surrounding bi-degree $\left(d_{1}, d_{2}\right)$ polynomials defined on the four faces are $p_{i, j}^{k}(x, y), k=0,1, \ldots, 3$ respectively (if the vertex $v_{i, j}$ is a T-junction, then some of the polynomials will be identical). For example for the left T-junction in Figure $2 \mathrm{~b}$, polynomials $p_{i, j}^{0}(x, y), p_{i, j}^{3}(x, y)$ are identical.

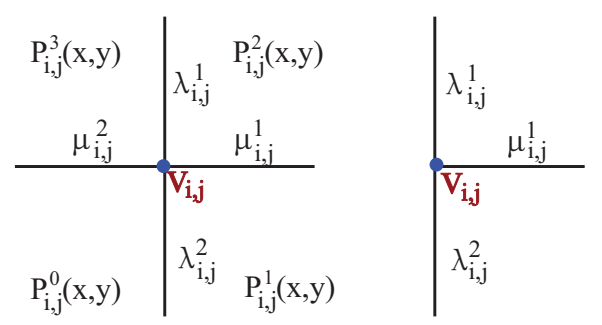

a

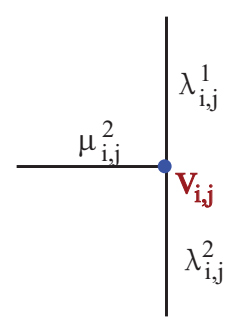

b

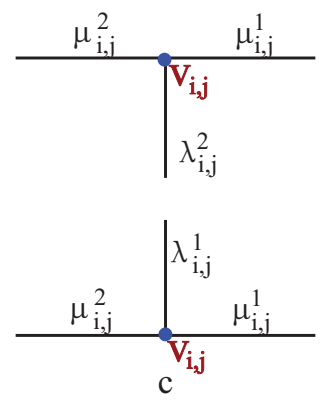

Figure 2: Smoothing cofactors around a vertex. 
Because $p_{i, j}^{0}(x, y)$ and $p_{i, j}^{1}(x, y)$ are $C^{\alpha}$ continuity, a bi-degree $\left(d_{1}-\alpha-1, d_{2}\right)$ polynomial $\lambda_{i, j}^{2}(y)$ exists such that

$$
p_{i, j}^{1}(x, y)-p_{i, j}^{0}(x, y)=\lambda_{i, j}^{2}(x, y)\left(x-x_{i}\right)^{\alpha+1},
$$

Here, $\lambda_{i, j}^{2}(x, y)=\sum_{p=0}^{d_{1}-\alpha-1} \sum_{q=0}^{d_{2}} e_{i, j}^{p, q}\left(x-x_{i}\right)^{p}\left(y-y_{j}\right)^{q}$ and the vector that contains the coefficients $e_{i, j}^{p, q}$ are called the edge cofactors for the common edge. If two polynomials are identical, then the edge cofactor is the zero vector.

Similarly, bi-degree $\left(d_{1}-\alpha-1, d_{2}\right)$ polynomial $\lambda_{i, j}^{1}(x, y)$, bi-degree $\left(d_{1}, d_{2}-\beta-1\right)$ polynomials $\mu_{i, j}^{1}(x, y)$ and $\mu_{i, j}^{2}(x, y)$ exist such that

$$
\begin{aligned}
& p_{i, j}^{2}(x, y)-p_{i, j}^{1}(x, y)=\mu_{i, j}^{1}(x, y)\left(y-y_{j}\right)^{\beta+1}, \\
& p_{i, j}^{3}(x, y)-p_{i, j}^{2}(x, y)=\lambda_{i, j}^{1}(x, y)\left(x-x_{i}\right)^{\alpha+1}, \\
& p_{i, j}^{0}(x, y)-p_{i, j}^{3}(x, y)=\mu_{i, j}^{2}(x, y)\left(y-y_{j}\right)^{\beta+1} .
\end{aligned}
$$

Summing all of these equations, we have

$$
\left(\lambda_{i, j}^{1}(x, y)+\lambda_{i, j}^{2}(x, y)\right)\left(x-x_{i}\right)^{\alpha+1}=-\left(\mu_{i, j}^{1}(x, y)+\mu_{i, j}^{2}(x, y)\right)\left(y-y_{j}\right)^{\beta+1} .
$$

Because $\left(x-x_{i}\right)^{\alpha+1}$ and $\left(y-y_{j}\right)^{\beta+1}$ are prime to each other, bi-degree $\left(d_{1}-\alpha-1, d_{2}-\beta-1\right)$ polynomial $d_{i, j}(x, y)$ exist such that,

$$
\begin{aligned}
& \lambda_{i, j}^{1}(x, y)+\lambda_{i, j}^{2}(x, y)=d_{i, j}(x, y)\left(y-y_{j}\right)^{\beta+1}, \\
& \mu_{i, j}^{1}(x, y)+\mu_{i, j}^{2}(x, y)=-d_{i, j}(x, y)\left(x-x_{i}\right)^{\alpha+1} .
\end{aligned}
$$

Let

$$
d_{i, j}(x, y)=\sum_{p=0}^{d_{1}-\alpha-1} \sum_{q=0}^{d_{2}-\beta-1} d_{i, j}^{p, q}\left(x-x_{i}\right)^{p}\left(y-y_{j}\right)^{q},
$$

We call $\hat{\mathbf{d}}_{i, j}$ the vertex cofactor, here $\hat{\mathbf{d}}_{i, j}$ is a vector that contains all of the coefficients $d_{i, j}^{p, q}$,

$$
\hat{\mathbf{d}}_{i, j}=\left(d_{i, j}^{0,0}, d_{i, j}^{0,1}, \ldots, d_{i, j}^{d_{1}-\alpha-1, d_{2}-\beta-2}, d_{i, j}^{d_{1}-\alpha-1, d_{2}-\beta-1}\right) .
$$

For boundary vertices, we only have part of the four equations. Therefore, we do not need to assign the bi-degree $\left(d_{1}-\alpha-1, d_{2}-\beta-1\right)$ polynomial $d_{i, j}(x, y)$ for the boundary vertex. Instead, we will assign some polynomials for the corresponding neighbor-boundary edge. For example, in Figure 3 a, we need two polynomials $\lambda_{i, j}^{1}$ and $\mu_{i, j}^{1}$ but for Figure $3 \mathrm{~b}$, we only need one polynomial $\mu_{i, j}^{1}$ because $\lambda_{i, j}^{1}=0$.

\subsection{Conformality conditions}

The vertex cofactors and edge cofactors are not totally free, there are other constraints for the continuity condition along each l-edge in the T-mesh.

We first consider a horizontal interior l-edge (referring to Figure 4) with $k+1$ vertices $\nu_{i_{t}, j}, t=$ $0,1, \ldots, k$. According to Equation 7 ,

$$
\begin{aligned}
\mu_{i_{0}, j}^{1}(x, y)+0 & =-d_{i_{0}, j}(x, y)\left(x-x_{i_{0}}\right)^{\alpha+1}, \\
\cdots & \\
\mu_{i_{t}, j}^{1}(x, y)+\mu_{i_{t}, j}^{2}(x, y) & =-d_{i_{t}, j}(x, y)\left(x-x_{i_{t}}\right)^{\alpha+1}, \\
\cdots & \\
0+\mu_{i_{k}, j}^{2}(x, y) & =-d_{i_{k}, j}(x, y)\left(x-x_{i_{k}}\right)^{\alpha+1} .
\end{aligned}
$$




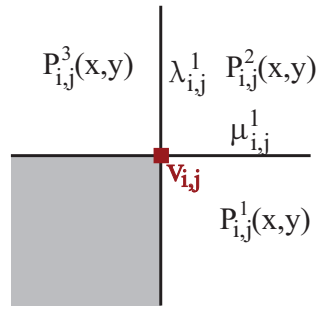

a.

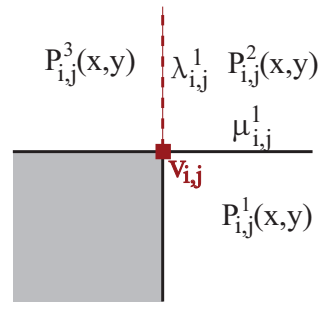

b.

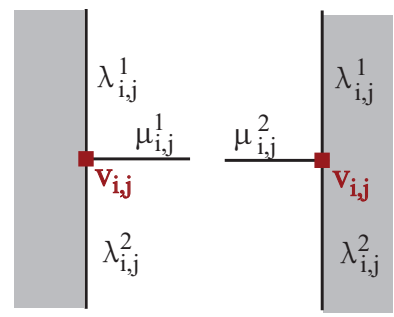

c.

Figure 3: Smoothing cofactors around a boundary vertex. The grey regions are outside of the T-mesh.

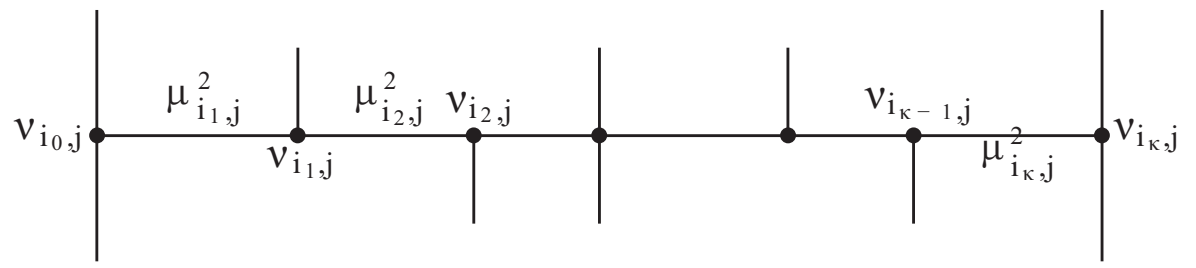

Figure 4: Smoothing cofactors along a horizontal edge segment.

and

$$
\mu_{i_{t}, j}^{2}(x, y)=-\mu_{i_{t-1}, j}^{1}(x, y)
$$

Summing all of these equations, we have the following equation:

$$
\sum_{t=0}^{k} d_{i_{t}, j}(x, y)\left(x-x_{i_{t}}\right)^{\alpha+1}=0 .
$$

Similarly, the constraints for a vertical interior l-edge are

$$
\sum_{t=0}^{l} d_{i, j_{t}}(x, y)\left(y-y_{j_{t}}\right)^{\beta+1}=0 .
$$

Now, we consider a horizontal ray with $r+1$ vertices $\nu_{i_{t}, j}, t=0,1, \ldots, r$. Without losing generality, we assume $\nu_{i_{0}, j}$ is a boundary vertex, then,

$$
\begin{aligned}
\mu_{i_{1}, j}^{1}(x, y)+\mu_{i_{1}, j}^{2}(x, y) & =-d_{i_{1}, j}(x, y)\left(x-x_{i_{1}}\right)^{\alpha+1}, \\
\ldots & \\
\mu_{i_{t}, j}^{1}(x, y)+\mu_{i_{t}, j}^{2}(x, y) & =-d_{i_{t}, j}(x, y)\left(x-x_{i_{t}}\right)^{\alpha+1} \\
\ldots & \\
0+\mu_{i_{r}, j}^{2}(x, y) & =-d_{i_{r}, j}(x, y)\left(x-x_{i_{r}}\right)^{\alpha+1}
\end{aligned}
$$

and

$$
\mu_{i_{r-1}, j}^{1}(x, y)=-\mu_{i_{r}, j}^{2}(x, y)
$$

Summing all of these equations, we have the following equation:

$$
\sum_{t=1}^{r} d_{i_{t}, j}(x, y)\left(x-x_{i_{t}}\right)^{\alpha+1}+\mu_{i_{1}, j}^{2}(x, y)=0 .
$$


A vertical ray has a similar equation except $x$ is changed to $y$. Additionally, we have an equation for a horizontal cross-cut (similar to a vertical cross-cut) with $c+1$ vertices $\nu_{i_{t}, j}, t=0,1, \ldots, c$, where

$$
\sum_{t=1}^{c-1} d_{i_{t}, j}(x, y)\left(x-x_{i_{t}}\right)^{\alpha+1}+\mu_{i_{1}, j}^{2}(x, y)+\mu_{i_{c}, j}^{1}(x, y)=0 .
$$

For each interior hole (referring to Figure 5), denote all of the polynomials for the surrounding horizontal neighbor-boundary edges $y=y_{i}$ to be $\mu_{i}(x, y), i=0,1, \ldots, h$, and the polynomials for the surrounding vertical neighbor-boundary edges $x=x_{j}$ to be $\lambda_{j}(x, y), j=0,1, \ldots, v$, then sum of all of the equations as (1), (2), (3), (4) and we have we have

$$
\sum_{i=0}^{h} \mu_{i}(x, y)\left(y-y_{i}\right)^{\beta+1}+\sum_{j=0}^{v} \lambda_{j}(x, y)\left(x-x_{j}\right)^{\alpha+1}=0 .
$$

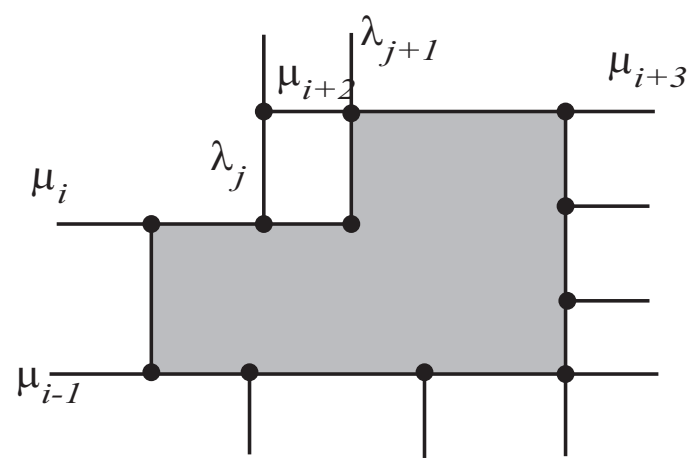

Figure 5: Smoothing cofactors around an interior hole (the grey region).

The linear systems (9), (10), (12), (13) and (14), which are associated with the interior l-edges, rays, cross-cuts and holes can be assembled into a global system as $\mathcal{F} \mathbf{y}=0$. Here, $\mathcal{F}$ is a matrix that is called the full conformality conditions matrix. Additionally, $\mathbf{y}$ is a column vector that has elements that include all of the vertex cofactors for the interior vertices and the coefficients for the polynomials for all of the neighbor-boundary edges in the T-mesh. According to [36], we have

Theorem 3.1. Given a $T$-mesh $\mathcal{T}$, let matrix $\mathcal{F}$ be the full conformality conditions matrix, then the dimension of the spline space over the T-mesh is,

$$
\operatorname{dim} \mathcal{S}\left(d_{1}, d_{2}, \alpha, \beta, \mathcal{T}\right)=\left(d_{1}+1\right)\left(d_{2}+1\right)+\operatorname{dim} \operatorname{ker}(\mathcal{F}) .
$$

If the T-mesh has no holes, then we can simplify the above linear systems into Theorem 3.3.

Lemma 3.2. Assuming that each $x_{i_{t}}$ and $y_{j_{t}}$ are distinct, then the dimensions of the solution space of equation (9) and (10) are $\left(d_{1}-\alpha\right)\left((k+1)\left(d_{2}-\beta\right)-d_{2}-1\right)_{+}$and $\left(d_{2}-\beta\right)\left((l+1)\left(d_{1}-\alpha\right)-d_{1}-1\right)_{+}$, respectively.

Proof. See [20] for more details. 
If the T-mesh has no holes, for a horizontal ray in the T-mesh as that in equation (12), for any vertex cofactors $d_{i_{t}, j}(x, y)$ assigned for the interior vertices, we can assign the polynomial $\mu_{i_{1}, j}^{2}(x, y)$ with $-\sum_{t=1}^{r} d_{i_{t}, j}(x, y)\left(x-x_{i_{t}}\right)^{\alpha+1}$ to satisfy the constraint. For a horizontal cross-cut in the T-mesh, because it has two boundary vertices, we can conclude that it has $\left(d_{1}+1\right)\left(d_{2}-\beta\right)$ degrees of freedom for any given vertex cofactors for the interior vertices. Similarly, a vertical cross-cut has $\left(d_{2}+1\right)\left(d_{1}-\alpha\right)$ degrees of freedom. Therefore, we can form a matrix as $\mathcal{M x}=0$ for the linear systems (9) or (10) that are associated with each interior l-edge. Here, $\mathcal{M}$ is a $n_{r} \times n_{c}$ matrix, which is called conformality conditions matrix. Additionally, $\mathbf{x}$ is a column vector whose elements are all of the vertex cofactors for the interior vertices in the T-mesh. According to the above analysis, we can obtain the dimension for the spline space over any general T-mesh without holes which is stated in the following theorem:

Theorem 3.3. Given a T-mesh $\mathcal{T}$ that has no holes, let matrix $\mathcal{M}$ be the conformality conditions matrix, then the dimension of the spline space over the T-mesh is,

$$
\begin{aligned}
\operatorname{dim} \mathcal{S}\left(d_{1}, d_{2}, \alpha, \beta, \mathcal{T}\right)= & \left(d_{1}+1\right)\left(d_{2}+1\right)+C^{h}\left(d_{1}+1\right)\left(d_{2}-\beta\right)+C^{v}\left(d_{2}+1\right) \\
& \left(d_{1}-\alpha\right)+V^{+}\left(d_{1}-\alpha\right)\left(d_{2}-\beta\right)+\operatorname{dim} \operatorname{ker}(\mathcal{M}),
\end{aligned}
$$

\section{Diagonalizable T-mesh}

Theorem 3.3 indicates that the main difficulty of computing the dimension of the spline space over a T-mesh is to calculate the rank of conformality condition matrix $\mathcal{M}$. It is obvious that the structure of the matrix is associated with the order of the edge conformality conditions and also the order of vertices cofactors. Most existing methods study the dimension of the matrix by forcing the T-mesh to be a nested structure, including those over the T-subdivision or hierarchical T-meshes. In the present paper, we find out a sufficient condition under which the dimension of spline space over a T-mesh without nested structure is stable.

We should mention that if the number of vertices in a horizontal l-edge is less than $N^{h}$ or the number of the vertices in a vertical l-edge is less than $N^{v}$, then the l-edge will not contribute the dimension of the spline space (we can delete the l-edge without altering the spline space). Thus, we called such an l-edge a vanished l-edge. Because the vanished l-edges cannot make much difference for the analysis, but it will make the dimension formula much more complex. Thus, for simplicity, in the following, we assume that all of the l-edges are not vanished l-edges.

Definition 4.1. Given a T-mesh $\mathcal{T}$, with ordered interior l-edges $e_{i_{j}}, j=1, \ldots, n_{e}$, then we can compute the new-vertex-vector $\nu^{i}$. Here $\nu_{1}^{i}$ is the number of vertices on l-edge $e_{i_{1}}$ and $\nu_{j}^{i}$ is the number of vertices on l-edge $e_{i_{j}}$ but not on l-edges $e_{i_{k}}, k<j$.

Definition 4.2. A T-mesh is called Diagonalizable if there exists an order of l-edges $e_{i_{j}}, j=$ $1,2, \ldots, n_{e}$ such that the new-vertex-vector $\nu^{i}$ satisfies that $\nu_{j}^{i} \geq N^{h}$ if $e_{i_{j}}$ is horizontal and $\nu_{j}^{i} \geq N^{v}$ if $e_{i_{j}}$ is vertical.

Lemma 4.3. If a T-mesh is diagonalizable, then the matrix $\mathcal{M}$ has full column rank if all the $x_{i}$ and $y_{j}$ are distinct.

Proof. Because the T-mesh is diagonalizable, there exists the order for the interior l-edges that satisfies the condition that is stated in definition 4.2. Without losing generality, we assume the order for the l-edges is $e_{i}, i=1,2, \ldots, n_{e}$. Then, we arrange the order of the edge conformality conditions that 
correspond to the l-edge from $e_{n_{e}}$ to $e_{1}$. The order of the vertex cofactors can be arranged in the following fashion: For l-edge $e_{n_{e}}$, if it is horizontal, there exist $N^{h}$ vertices that do not appear in the other l-edges. Each vertex corresponds to $\left(d_{1}-\alpha\right) \times\left(d_{2}-\beta\right)$ cofactors, so these $N^{h}$ vertices correspond to $\left(d_{1}-\alpha\right) \times\left(d_{2}-\beta\right) N^{h} \geq\left(d_{1}+1\right)\left(d_{2}-\beta\right)$ cofactors. There exist $\left(d_{1}+1\right)\left(d_{2}-\beta\right)$ cofactors such that the corresponding matrix block has full rank, and we put these cofactors in the beginning of $\mathbf{x}^{T}$. If the l-edge is vertical, similarly we can select $\left(d_{2}+1\right)\left(d_{1}-\alpha\right)$ cofactors such that the corresponding matrix block has full rank. Then we put them in the next of $\mathbf{x}^{T}$. This process can be applied for the remaining l-edges. After the arrangement, an appropriate partition of the linear system of constraints is

$$
\left[\mathcal{M}_{1} \mid \mathcal{M}_{2}\right]\left[\frac{\mathbf{x}_{1}}{\mathbf{x}_{2}}\right]=\mathbf{0}
$$

where $\mathcal{M}_{1}$ is a $n_{r} \times n_{r}$ matrix, $\mathcal{M}_{2}$ is a $n_{r} \times\left(n_{c}-n_{r}\right)$ matrix, $\mathbf{x}_{1}^{T}$ is a vector of the first $n_{r}$ vertex cofactors, and $\mathbf{x}_{2}$ is a vector of the remaining vertex cofactors.

Because the matrix $\mathcal{M}_{1}$ is in upper block triangular form and according to Lemma 3.2 each diagonal block $\left(d_{2}-\beta\right)\left(d_{1}+1\right) \times\left(d_{2}-\beta\right)\left(d_{1}+1\right)$ or $\left(d_{1}-\alpha\right)\left(d_{2}+1\right) \times\left(d_{1}-\alpha\right)\left(d_{2}+1\right)$ matrix is full rank, thus matrix $\mathcal{M}_{1}$ is obviously of full rank.

Theorem 4.4. Suppose $T$-mesh $\mathcal{T}$ is diagonalizable and has no vanished l-edges and holes, then the dimension of the spline space over the T-mesh is,

$$
\begin{aligned}
\operatorname{dim} \mathcal{S}\left(d_{1}, d_{2}, \alpha, \beta, \mathcal{T}\right)= & \left(d_{1}+1\right)\left(d_{2}+1\right)+\left(C^{h}-T^{h}\right)\left(d_{1}+1\right)\left(d_{2}-\beta\right)+ \\
& \left(C^{v}-T^{v}\right)\left(d_{2}+1\right)\left(d_{1}-\alpha\right)+V\left(d_{1}-\alpha\right)\left(d_{2}-\beta\right) .
\end{aligned}
$$

Proof. Because T-mesh $\mathcal{T}$ is diagonalizable, $\operatorname{dim} \operatorname{ker}(\mathcal{M})$ is

$$
n_{c}-n_{r}=\left(d_{1}-\alpha\right)\left(d_{2}-\beta\right)\left(V-V^{+}\right)-\left(d_{1}+1\right)\left(d_{2}-\beta\right) T^{h}-\left(d_{2}+1\right)\left(d_{1}-\alpha\right) T^{v} .
$$

According to theorem 3.3, we have the dimension $\operatorname{dim} \mathcal{S}\left(d_{1}, d_{2}, \alpha, \beta, \mathcal{T}\right)$ that is

$$
\begin{aligned}
\operatorname{dim}= & \left(d_{1}+1\right)\left(d_{2}+1\right)+C^{h}\left(d_{1}+1\right)\left(d_{2}-\beta\right)+C^{v}\left(d_{2}+1\right)\left(d_{1}-\alpha\right)+ \\
& V^{+}\left(d_{1}-\alpha\right)\left(d_{2}-\beta\right)+\left(d_{1}-\alpha\right)\left(d_{2}-\beta\right)\left(V-V^{+}\right)- \\
& \left(d_{1}+1\right)\left(d_{2}-\beta\right) T^{h}-\left(d_{2}+1\right)\left(d_{1}-\alpha\right) T^{v} \\
= & \left(d_{1}+1\right)\left(d_{2}+1\right)+\left(C^{h}-T^{h}\right)\left(d_{1}+1\right)\left(d_{2}-\beta\right)+\left(C^{v}-T^{v}\right) \\
& \left(d_{2}+1\right)\left(d_{1}-\alpha\right)+V\left(d_{1}-\alpha\right)\left(d_{2}-\beta\right) .
\end{aligned}
$$

Theorem 4.5. A necessary and sufficient condition for a T-mesh to be diagonalizable is for any interior l-edges set $\mathbb{S}$, there exists at least one horizontal l-edge such that the number of vertices on this l-edge but not on the other l-edges in $\mathbb{S}$ is at least $N^{h}$, or there exists at least one vertical l-edge such that the number of vertices on this l-edge but not on the other l-edges in $\mathbb{S}$ is at least $N^{v}$.

Proof. First, we prove the condition is necessary by reducing absurdity. If the T-mesh is diagonalizable, there exists a set of l-edges $\left\{e_{i_{1}}, e_{i_{2}}, \ldots, e_{i_{s}}\right\}$ such that any horizontal l-edge in the set has at most $N^{h}-1$ vertices, which are not on the other l-edges in the set and that any vertical l-edge has at most $N^{v}-1$ vertices, which are not on the other l-edges in the set. Because the T-mesh is diagonalizable, without losing of generality, we assume that when the order of the interior l-edges is $e_{1}, e_{2}, \ldots, e_{n_{e}}$, 
which satisfies the condition of diagonalizable. Let $k$ be the maximal index for all $i_{j}, j=1, \ldots, s$. Now, we consider l-edge $e_{k}$. Because it has at most $N^{h}-1$ or $N^{v}-1$ vertices, which are not on the other l-edges in the set, it also has at most $N^{h}-1$ or $N^{v}-1$ vertices, which are not on the l-edges for $e_{1}, \ldots, e_{k-1}$ because $\mathbb{S} \subseteq\left\{e_{1}, \ldots, e_{k-1}, e_{k}\right\}$, which violates the assumption of diagonalizable. Thus, the condition is necessary.

Now, we prove that the condition is sufficient. For the set of l-edges $e_{i}, i=1, \ldots, n_{e}$, according to the assumption, there exists one l-edge that has a sufficient amount of vertices on the l-edge but not on the other l-edges. Without losing generality, we assume it is $e_{1}$. Suppose we have ordered the l-edges as $e_{1}, e_{2}, \ldots, e_{j}$ to satisfy the diagonalizable condition. Then for set $\left\{e_{j+1}, \ldots, e_{n_{e}}\right\}$, according to the assumption, there exists one l-edge which has a sufficient amount of vertices on the l-edge but not on the other l-edges. Without losing generality, we assume it is $e_{j+1}$. By using this process, we can order the l-edges as $e_{j}, j=n_{e}, n_{e}-1, \ldots, 1$ which satisfies the diagonalizable condition.

Now we provide some examples for dimension of the spline space over the following T-meshes.
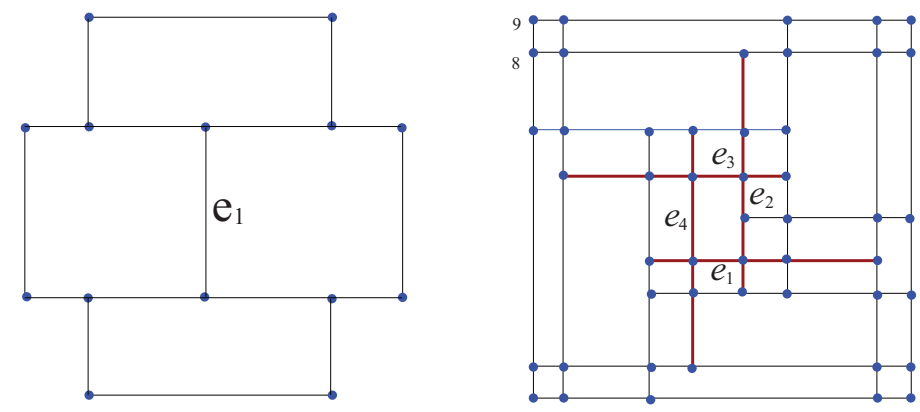

Figure 6: Two example T-meshes for spline space $\mathcal{S}(3,3,2,2, \mathcal{T})$.

Example 4.1. We first consider the spline space $\mathcal{S}(3,3,2,2, \mathcal{T})$ over the two T-meshes in Figure 6.

The first T-mesh has a few concave corners and one interior l-edge which is a vanished l-edge because it has only two vertices. Additionally the T-mesh has two cross-cut. Therefore, according to theorem 3.3, the dimension of the spline space $\mathcal{S}(3,3,2,2, \mathcal{T})$ over the first $T$-mesh is $16+2 \times 4=24$.

The second T-mesh has four interior l-edges $e_{i}, i=1, \ldots, 4$, four cross-cuts and five rays. According to the definition of the diagonalizable T-mesh (the order is $e_{4}, e_{1}, e_{3}, e_{2}$ ) or by using theorem 4.5, we can easily see that the T-mesh is diagonalizable. In the T-mesh, we have 4 cross-cuts, 2 horizontal interior l-edges, 2 vertical interior l-edges and 31 interior vertices. Therefore according to theorem 4.4, the dimension of the spline space $\mathcal{S}(3,3,2,2, \mathcal{T})$ over the $T$-mesh is $16+4(4-4)+31=47$. We should mention that the T-mesh is not a T-subdivision or a hierarchical T-mesh.

Example 4.2. We now consider the spline space $\mathcal{S}(3,3,1,1, \mathcal{T})$ over the two T-meshes in Figure 7.

The first T-mesh has four interior l-edges. Additionally we can see that it is not diagonalizable because the new-vertex-vector could be $(3,2,2,1)$ or $(3,3,1,1)$ for different orders. Thus, we cannot complete the dimension by using the current method. Actually, according to our knowledge, no existing method can compute the dimension of such a spline space.

The second T-mesh also has four interior l-edges, if we arrange the order of the l-edges to be $e_{1}$, $e_{2}, e_{3}$ and $e_{4}$, we can see that the new-vertex-vector is $(3,2,2,2)$ (i.e., the T-mesh is diagonalizable). 

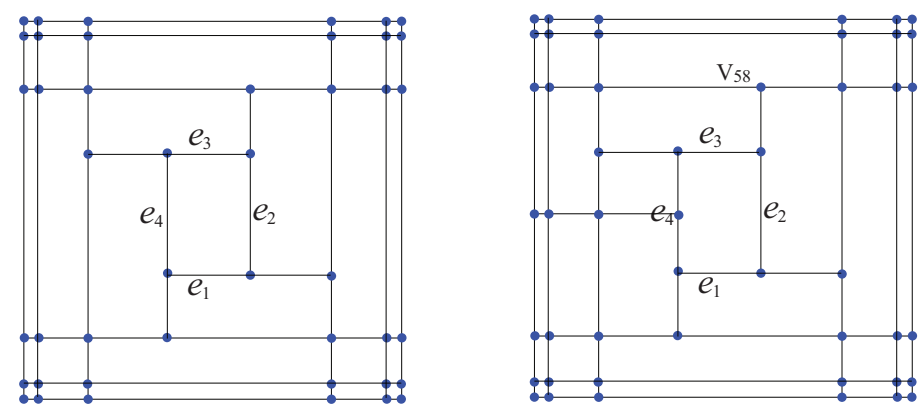

Figure 7: Non-diagonalizable vs. diagonalizable for spline space $\mathcal{S}(3,3,1,1, \mathcal{T})$.

Because the T-mesh has 8 cross-cuts, 4 interior l-edges, and 27 interior vertices, the dimension of the spline space $\mathcal{S}(3,3,1,1, \mathcal{T})$ over the second T-mesh is $16+4 \times 2 \times(8-4)+27 \times 4=156$.

A diagonalizable T-mesh can have l-cycles (e.g., the right T-mesh in Figure 7, the T-meshes that satisfy the conditions in Theorem 5.1 and extended analysis-suitable T-meshes in [18]). However, we cannot conclude that a T-mesh without c-cycles is a diagonalizable T-mesh, which is shown in the counterexample that is illustrated in Figure 8. This means that certain T-meshes exist that can be handled by [10] and [19] but cannot be handled by the methods based on the smoothing cofactorconformality method and the homological technique.

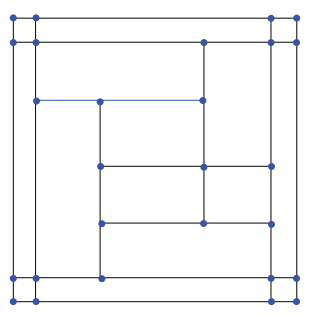

Figure 8: The T-mesh has no c-cycles, but has an l-cycle. And it is also not a diagonalizable T-mesh for spline space $\mathcal{S}(3,3,1,1, \mathcal{T})$.

\section{Application}

In this section, we will provide new dimension results for the spline space over T-meshes without nested structures. A similar result is also got in [38] independently from the present paper. But based on the diagonalizable T-meshes, we can have a simple proof.

Theorem 5.1. Given a regular $T$-mesh $\mathcal{T}$ without holes, if each horizontal l-edges has at least $N^{h}-1$ mono-vertices and each vertical l-edge has at least $N^{v}-1$ mono-vertices besides the two end vertices, then the dimension of the spline space over $\mathcal{T}$ is

$$
\begin{aligned}
\operatorname{dim} \mathcal{S}\left(d_{1}, d_{2}, \alpha, \beta, \mathcal{T}\right)= & \left(d_{1}+1\right)\left(d_{2}+1\right)+\left(C^{h}-T^{h}\right)\left(d_{1}+1\right)\left(d_{2}-\beta\right)+ \\
& \left(C^{v}-T^{v}\right)\left(d_{2}+1\right)\left(d_{1}-\alpha\right)+V\left(d_{1}-\alpha\right)\left(d_{2}-\beta\right) .
\end{aligned}
$$




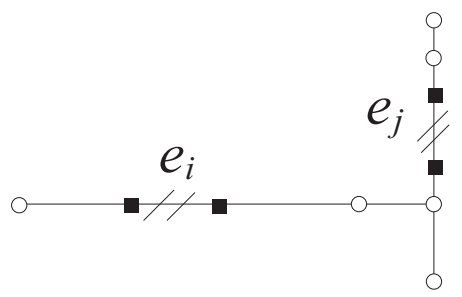

Figure 9: The new correction theorem for [20].

Proof. We will prove that the T-mesh is diagonalizable under the condition by reducing absurdity. If the T-mesh is not diagonalizable, referring to Figure 9, for any set of l-edges $\left\{e_{1}, \ldots, e_{n}\right\}$, if all the l-edges are vertical l-edges, then for any vertical l-edge in the set, it at least has $N^{v}$ vertices which are not on the other l-edges in the set which violates the Theorem 4.5. Otherwise, suppose $e_{i}$ is the most bottom horizontal l-edge in the set (if there is more than one l-edge in the set, we will pick the leftmost one). According to the assumption, $e_{i}$ has at least $N^{h}-1$ mono-vertices (black rectangle vertices in the figure), if one of the two end vertices is not on the other l-edges in the set, then $e_{i}$ at least has $N^{h}$ vertices which are not on the other l-edges in the set. However, this violates the Theorem 4.5. Thus, there exists a vertical l-edge which contains one of the end vertices of $e_{i}$. Without losing generality, we assume the right end vertex is on the l-edge $e_{j}$ in the set. Now, we consider l-edge $e_{j}$, the bottom end vertex of the l-edge cannot lie on the other l-edges in the set because it is on the bottom of $e_{i}$ which is the bottommost l-edge in the set. Therefore, $e_{j}$ at least has $N^{v}$ vertices which are not on the other l-edges in the set which violates the Theorem 4.5. Thus, the T-mesh satisfies the condition in the lemma is a diagonalizable T-mesh. By using Theorem 4.4, we prove the theorem.

\section{Discussion and future work}

In this paper, we introduce a class of T-meshes (diagonalizable T-meshes), over which the dimension of spline spaces is stable. We also provide a necessary and sufficient condition that is used to characterize this class of T-meshes. By using the tools, we provide new dimension results for the spline space over the T-meshes without nested structures.

The paper leaves several problems for the future research. Because we have provided the dimension of the spline space, there are many problems that need to be solved. For examples, how to construct a set of basis functions with good properties, how to apply the spline to geometric modeling and isogeometric analysis, etc. Also, it is an important and interesting question to find out other class of spline space with stable dimension. We will explore all of these problems in the future.

\section{Acknowledgements}

This work was supported by the NSF of China (No.11031007, No.60903148), the Chinese Universities Scientific Fund, SRF for ROCS SE, the CAS Startup Scientific Research Foundation and NBRPC 2011CB302400.

\section{References}

[1] G. Farin, NURBS Curves and Surfaces: from Projective Geometry to Practical Use, Fourht Edition, A. K. Peters, Ltd., Natick, MA, 2002. 
[2] J. A. Cottrell, T. J. R. Hughes, Y. Bazilevs, Isogeometric analysis: Toward Integration of CAD and FEA, Wiley, Chichester, 2009.

[3] D. Forsey, R. Bartels, Hierarchical B-spline refinement, Comput. Graph. 22 (1988) 205-212.

[4] R. Kraft, Adaptive and linearly independent multilevel B-splines, in: L. Mehauté, C. A., Rabut, L. Schumaker (Eds.), Surface Fitting and Multiresolution Methods, Vanderbilt University Press, 1997, pp. 209-218.

[5] A.-V. Vuong, C. Giannelli, B. Jüttler, B. Simeon, A hierarchical approach to adaptive local refinement in isogeometric analysis, Comput. Methods Appl. Mech. Engrg. 200 (2011) 3554-3567.

[6] C. Giannelli, B. Jüttler, H. Speleers, THB-splines: The truncated basis for hierarchical splines, Computer Aided Geometric Design 29 (2012) 485-498.

[7] T. W. Sederberg, J. Zheng, A. Bakenov, A. Nasri, T-splines and T-NURCCSs, ACM Transactions on Graphics 22 (3) (2003) 477-484.

[8] T. W. Sederberg, D. L. Cardon, G. T. Finnigan, N. S. North, J. Zheng, T. Lyche, T-spline simplification and local refinement, ACM Transactions on Graphics 23 (3) (2004) 276-283.

[9] X. Li, J. Zheng, T. W. Sederberg, T. J. R. Hughes, M. A. Scott, On the linear independence of T-splines blending functions, Computer Aided Geometric Design, 29 (2012) 63-76.

[10] J. Deng, F. Chen, Y. Feng, Dimensions of spline spaces over T-meshes, Journal of Computational and Applied Mathematics 194 (2006) 267-283.

[11] J. Deng, F. Chen, X. Li, C. Hu, W. Tong, Z. Yang, Y. Feng, Polynomial splines over hierarchical T-meshes, Graphical Models 74 (2008) 76-86.

[12] T. Dokken, T. Lyche, K. F. Pettersen, Polynomial splines over locally refined box-partitions, Computer Aided Geometric Design 30 (2013) 331-356.

[13] B. Mourrain, On the dimension of spline spaces on planar T-meshes, Math. Comp. 83 (2014) $847-871$.

[14] Y. Bazilevs, V. M. Calo, J. A. Cottrell, J. A. Evans, T. J. R. Hughes, S. Lipton, M. A. Scott, T. W. Sederberg, Isogeometric analysis using T-splines, Computer Methods in Applied Mechanics and Engineering 199 (5-8) (2010) 229 - 263.

[15] Y. Bazilevs, L. Beirao de Veiga, J. Cottrell, T. Hughes, G. Sangalli, Isogeometric analysis: approximation, stability and error estimates for $h$-refined meshes, Mathematical Models and Methods in Applied Sciences 16 (2006) 1031-1090.

[16] C. Giannelli, B. Jüttler, Bases and dimensions of hierarchical tensor-product spline spaces, Journal of Computational and Applied Mathematics 239 (2013) 162-178.

[17] M. A. Scott, X. Li, T. W. Sederberg, T. J. R. Hughes, Local refinement of analysis-suitable Tsplines, Computer Methods in Applied Mechanics and Engineering 213-216 (2012) 206-222.

[18] X. Li, M. A. Scott, Analysis-suitable T-splines: Characterization, refinablility and approximation, Mathematical Models and Methods in Applied Sciences 24(6) (2014) 1141-1164. 
[19] L. L. Schumaker, L. Wang, Spline spaces on TR-meshes with hanging vertices, Numerische Mathematik 118 (2011) 531-548.

[20] C. J. Li, R. H. Wang, F. Zhang, Improvement on the Dimensions of Spline Spaces on T-Mesh, Journal of Information \& Computational Science 3 (2) (2006) 235-244.

[21] L. L. Schumaker, L. Wang, Approximation power of polynomial splines on T-meshes, Computer Aided Geometric Design 29 (2012) 599-612.

[22] C.-J. Li, J. Chen, On the dimensions of bivariate spline spaces and the stability of the dimensions, Journal of Computational and Applied Mathematics 236 (2011) 765-774.

[23] T. W. Sederberg, G. T. Finnigan, X. Li, H. Lin, H. Ipson, Watertight trimmed NURBS, ACM Transactions on Graphics 27 (3) (2008) Article no. 79.

[24] X. Li, J. Deng, F. Chen, Surface modeling with polynomial splines over hierarchical T-meshes, The Visual Computer 23 (2007) 1027-1033.

[25] X. Li, J. Deng, F. Chen, Polynomial splines over general T-meshes, The Visual Computer 26 (2010) $277-286$.

[26] N. Nguyen-Thanh, H. Nguyen-Xuan, S. P. A. Bordas, T. Rabczuk, Isogeometric analysis using polynomial splines over hierarchical T-meshes for two-dimensional elastic solids, Computer Methods in Applied Mechanics and Engineering 200 (2011) 1892-1908.

[27] P. Wang, J. Xu, J. Deng, F. Chen, Adaptive isogeometric analysis using rational PHT-splines, Computer-Aided Design 43 (2011) 1438-1448.

[28] L. Tian, F. Chen, Q. Du, Adaptive finite element methods for elliptic equations over hierarchical T-meshes, J. Comput. Appl. Math. 236 (2011) 878-891.

[29] L. L. Schumaker, L. Wang, Splines on triangulations with hanging vertices, Constructive Approximation 36 (2012) 487-511.

[30] A. Buffa, D. Cho, M. Kumar, Characterization of T-splines with reduced continuity order on Tmeshes, Comput. Methods Appl. Mech. Engrg. 201-204 (2012) 112-126.

[31] X. Li, F. Chen, On the instability in the dimension of spline space over particular T-meshes, Computer Aided Geometric Design 28 (2011) 420-426.

[32] D. Berdinsky, M. Oh, T. Kim, B. Mourrain, On the problem of instability in the dimension of a spline space over a T-mesh, Computers Graphics 36(2) (2012) 507-513.

[33] M. Wu, J. Deng, F. Chen, The dimension of spline spaces with highest order smoothness over hierarchical T-meshes, Computer Aided Geometric Design 30 (2013) 20-34.

[34] J. Deng, F. Chen, L. Jin, Dimensions of biquadratic spline spaces over T-meshes, Journal of Computational and Applied Mathematics 238 (2013) 68-94.

[35] C. Zeng, F. Deng, X. Li, J. Deng, Dimensions of biquadratic and bicubic spline spaces over hierarchical T-meshes, Journal of Computational and Applied Mathematics 287 (2015) 162-178. 
[36] R.-H. Wang, Multivariate Spline Functions and Their Applications, Science Press/ Kluwer Academic Publishers, 2001.

[37] L. Schumaker, On the dimension of spaces of piecewise polynomials in two variables, in: Multivariate Approximation Theory, In: Schempp, W., Zeller, K. (Eds.), Birkhauser Verlag, Basel, 1979, pp. 396-412.

[38] Q. Guo, R. Wang, C. Li, On the problem of instability in the dimensions of spline spaces over T-meshes with T-cycles, J. Comp. Math. 33 (2015) 248-262. 